\title{
Reconstruction-Independent 3D CAD for Calcification Detection in Digital Breast Tomosynthesis Using Fuzzy Particles
}

\author{
G. Peters ${ }^{1,3}$, S. Muller ${ }^{1}$, S. Bernard ${ }^{1}$, R. Iordache ${ }^{1}$, \\ F. Wheeler ${ }^{2}$, and I. Bloch ${ }^{3}$ \\ ${ }^{1}$ GE Healthcare Europe, 283, rue de la Minière, 78533 Buc, France \\ \{gero.peters, serge.muller, sylvain.bernard, \\ razvan.iordache\} @med.ge.com \\ ${ }^{2}$ GE Global Research, One Research Circle, Niskayuna, NY 12309, USA \\ wheeler@research.ge.com \\ ${ }^{3}$ Ecole Nationale Supérieure de Télécommunications, \\ CNRS UMR 5141 LTCI, Paris, France \\ isabelle.bloch@enst.fr
}

\begin{abstract}
In this paper we present a novel approach for microcalcification detection in Digital Breast Tomosynthesis (DBT) datasets. A reconstructionindependent approach, working directly on the projected views, is proposed. Wavelet filter responses on the projections are thresholded and combined to obtain candidate microcalcifications. For each candidate, we create a fuzzy contour through a multi-level thresholding process. We introduce a fuzzy set definition for the class microcalcification contour that allows the computation of fuzzy membership values for each candidate contour. Then, an aggregation operator is presented that combines information over the complete set of projected views, resulting in 3D fuzzy particles. A final decision is made taking into account information acquired over a range of successive processing steps. A clinical example is provided that illustrates our approach. DBT still being a new modality, a similar published approach is not available for comparison and limited clinical data currently prevents a clinical evaluation of the algorithm. .
\end{abstract}

\section{Introduction}

Breast cancer continues to be one of the leading causes of cancer mortality among women. Since the underlying causes for this disease remain unknown, early screening is the only means to reduce mortality among the affected population. X-ray mammography is currently the primary method for detecting early breast cancers, reducing the mortality rate by about $30 \%$ for women 50 years and older [1]. However, about $30 \%$ of breast cancers are still missed by conventional screening mammography. One of the main reasons is the superimposition of tissue that obscures lesions in dense breasts [2]. Digital Breast Tomosynthesis (DBT) [3],[4], is a new three-dimensional (3D) limited-angle tomography breast imaging technique that will substantially overcome the superimposition problem for lesion detection. It then remains important to accu- 
rately detect and localize microcalcification clusters, which are one of the earliest signs of breast cancer visible in mammograms.

The introduction of DBT brings a variety of new challenges and benefits. Several projected views from different acquisition angles will potentially reduce the number of false positives (FP) caused by summation artifacts as well as the number of false negatives (FN) caused by the masking effect of overlying tissue. At the same time, the dose per acquired image is significantly reduced in comparison to standard 2D mammograms, to maintain a comparable total patient dose per scan. This has a major impact on any processing in the projections, as the characteristics of these images change dramatically, and algorithms developed for 2D mammograms cannot be generally applied without modification.

As DBT systems become available for clinical testing, different strategies for CAD on DBT data are emerging. Chan et al. have presented an approach applying CAD processing on reconstructed slices [6]. A method applying mass detection algorithms directly on the projected views was presented in [7]. Candidates are detected in each projected view separately and afterwards combined in 3D using the acquisition geometry. CAD processing for calcification detection in 3D DBT data has not been made public so far and therefore represents one of the original contributions of this paper. Since DBT is a relatively new modality, 3D reconstruction algorithms for its particular geometry are still not fully optimized. Hence, it is desirable to devise a $\mathrm{CAD}$ approach that is independent of the reconstruction algorithm used to generate tomosynthesis slices.

Fuzzy processing has been widely accepted for use in microcalcification detection tasks [8], [9], [10]. In the present work, we propose an original method using fuzzy particles to account for ambiguities in shape and appearance of microcalcifications for the purpose of modeling and identification. The use of a fuzzy set description enables us to maintain the evidence, and the strength of the evidence, gathered from each DBT projection image for each potential finding without making hard decisions in isolation. The final decision as to the presence or absence of calcification is then made in 3D through aggregation of all available information from all projections.

Working directly on DBT projected views offers several advantages. The processing time is reduced compared to the processing of reconstructed slices since they are generally much more numerous than the projected views. The processing is performed on a data space independent of the reconstruction algorithm used to generate 3D images. There are however some issues that need to be addressed. The DBT projected views have a lower Contrast to Noise Ratio (CNR) rendering the detection task in a single image more difficult when using approaches designed for conventional 2D mammograms. It is crucial to delay the detection decision for each candidate particle until information from each view can be jointly considered. With this as our motivation, we develop and present a fuzzy processing operator that aggregates the information extracted from each projected view.

Low-dose projected views contain ambiguities about the objects in the image, including uncertainty about a candidate particle being a microcalcification, imprecision of its position and extent, as well as the incomplete nature of data in the individual projections. We use fuzzy logic to take these ambiguities into account and preserve 
them up to a point in the processing where we have gathered sufficient information to make a decision that simultaneously utilizes all available information.

The novel approach presented here consists of the following processing steps. We start by detecting candidate particles that potentially are microcalcifications. We then build a fuzzy contour for each candidate particle, based on several extracted attributes and multi-level segmentation. A partial defuzzification is applied, resulting in fuzzy particles better suited for the final aggregation operation. Once information from the entire set of projected views has been aggregated resulting in 3D fuzzy particles, their properties are extracted before the final step deciding whether those particles correspond to microcalcifications or other structures.

\section{Candidate Particle Detection}

In the initial processing performed on the projected views we extract a map of candidate particles. A "Mexican Hat" wavelet kernel is used to compute the contrast between a structure located in the center of the wavelet and its surrounding neighborhood. Convolving the original image with this kernel creates a band-pass image of sorts that emphasizes small structures in the image. Our implementation incorporates a multi-scale approach to account for the range in size of microcalcifications. The images resulting from the application of wavelets at different scales are combined using a "max" operator resulting in a local contrast image. This image is thresholded against local variance of background noise level. The connected components of this binary image are then labeled as candidates.

This initial step is crucial to all further processing. Any particle missed by the initial detection cannot be recovered later on. A high sensitivity in this step is therefore of utmost importance. To achieve the desired sensitivity we accept an elevated number of false positives (FP), which we will be able to reduce at a later stage with the use of fuzzy particles and the aggregation of information from different projected views.

\section{2D Fuzzy Contours}

Once the candidate particles have been identified, we create fuzzy contours describing each candidate. Each fuzzy contour accounts for the ambiguities of the original data. For each candidate particle, we compute a set of contour candidates using multi-level thresholding. This ordered set of contours is considered the universe of all possible contours describing a given particle. The prior knowledge about contours of microcalcifications [5] is transformed into a fuzzy set description. Finally, membership values for each contour candidate are calculated.

First, we extract a set of candidate contours for each candidate particle using multilevel thresholding. This is achieved by applying a series of decreasing thresholds to the local contrast image and extracting the level-set. Each candidate particle is treated separately. This processing is applied until either one of the conditions given in Equations (1) and (2) is met 


$$
A(C) \leq A_{\max }
$$

where $A(C)$ is the area enclosed by the contour $C$ and $A_{\max }$ is the maximum expected size for the area of a microcalcification

$$
G(\rho, C) \geq G(\rho)_{\max }-\Delta G_{\max }
$$

where $G(\rho, C)$ is the pixel intensity under the contour $C$ of particle $\rho, G(\rho)_{\max }$ is the maximum pixel intensity of particle $\rho$, and $\Delta G_{\max }$ is the intensity range expected within a single microcalcification. These two conditions limit the area and intensity range of the candidate particles being extracted to values consistent with actual microcalcifications.

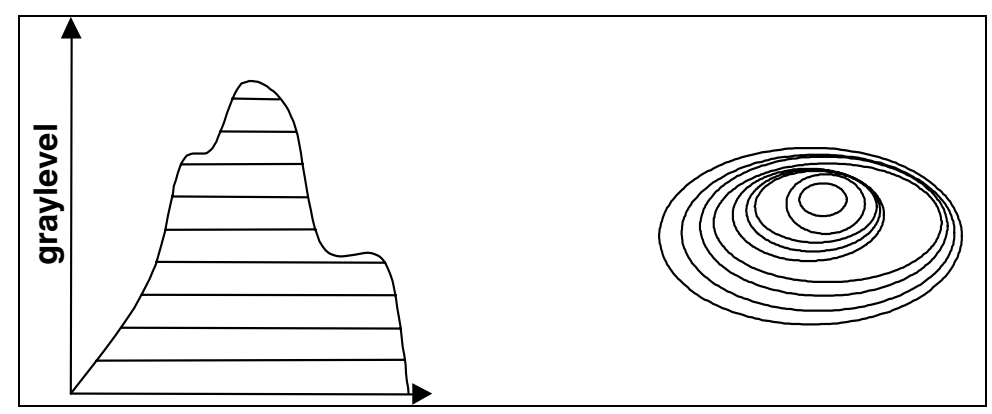

Fig. 1. The function on the left shows a gray level profile of a candidate particle and the thresholds applied. The corresponding extracted contours are shown on the right.

For each candidate particle, we thus obtain a set of candidate contours $\left\{C_{i}\right\}$. In order to create a fuzzy contour we compute, for each contour $C_{i}$, the membership value $f_{c}\left(C_{i}\right)$ to the microcalcification contour class.

The fuzzy set corresponding to this class is defined based on prior knowledge about characteristics of microcalcifications, which is summarized by "microcalcifications are small and have a high contrast". This linguistic definition translates to a fuzzy set description using two attributes namely area and gradient shown in Fig. 2.

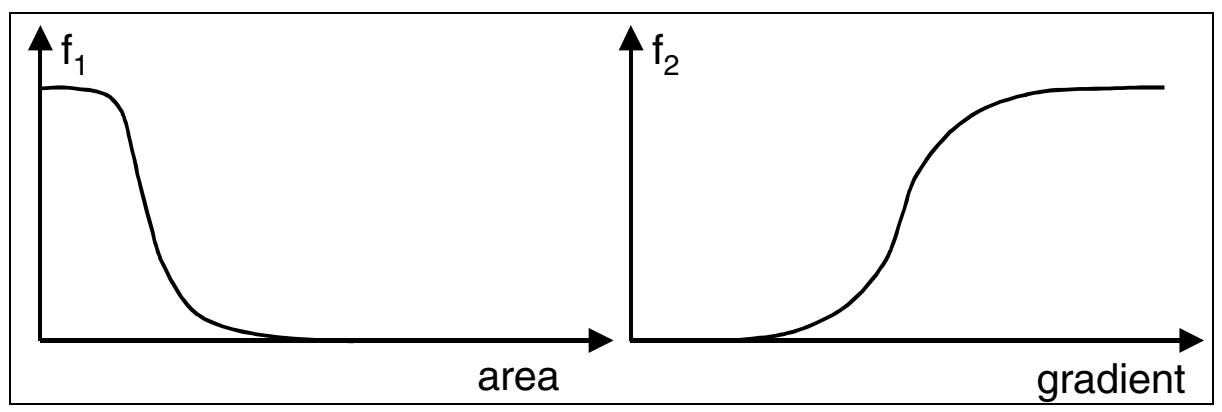

Fig. 2. The above functions correspond to fuzzy set representations of size (size is small) and image gradient under a contour (gradient is high) for particles in the mammography images 
The functions depicted in Fig. 2 correspond to fuzzy set representations of size (size is small) and image gradient under a contour (gradient is high). The functions have been designed experimentally in prior work [9].

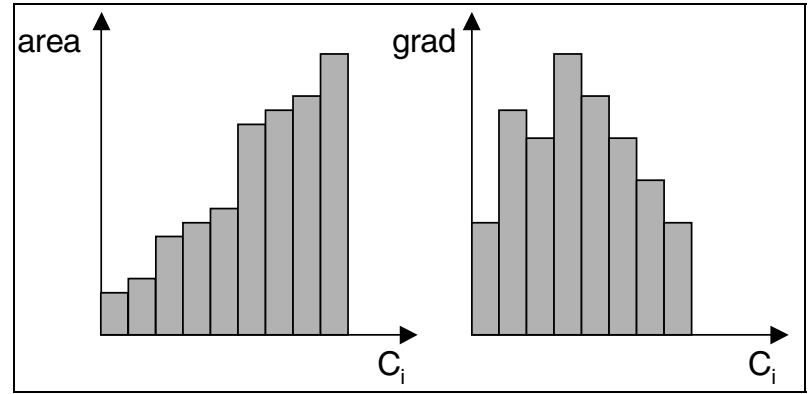

Fig. 3. Values for the fuzzy contour of a given candidate particle for area (left) and gradient under the contour (right)

For each candidate contour $C_{i}$, we measure both area $A$ and gradient $g$ values (Fig. 3). We can derive the membership values $f_{\text {area }}\left(C_{i}\right)$ and $f_{\text {gradient }}\left(C_{i}\right)$ for each contour based on small area and high gradient criteria as

$$
f_{\text {area }}\left(C_{i}\right)=f_{1}\left(A\left(C_{i}\right)\right) ; \quad f_{\text {gradient }}\left(C_{i}\right)=f_{2}\left(g\left(C_{i}\right)\right)
$$

The conjunction of membership values obtained for each contour based on small area and high gradient provides membership values $f_{c}\left(C_{i}\right)$ to the class microcalcification contour [9],

$$
f_{c}\left(C_{i}\right)=\wedge\left[f_{\text {area }}\left(C_{i}\right), f_{\text {gradient }}\left(C_{i}\right)\right]
$$

Application of this method for one particular candidate particle is shown in Fig. 4.

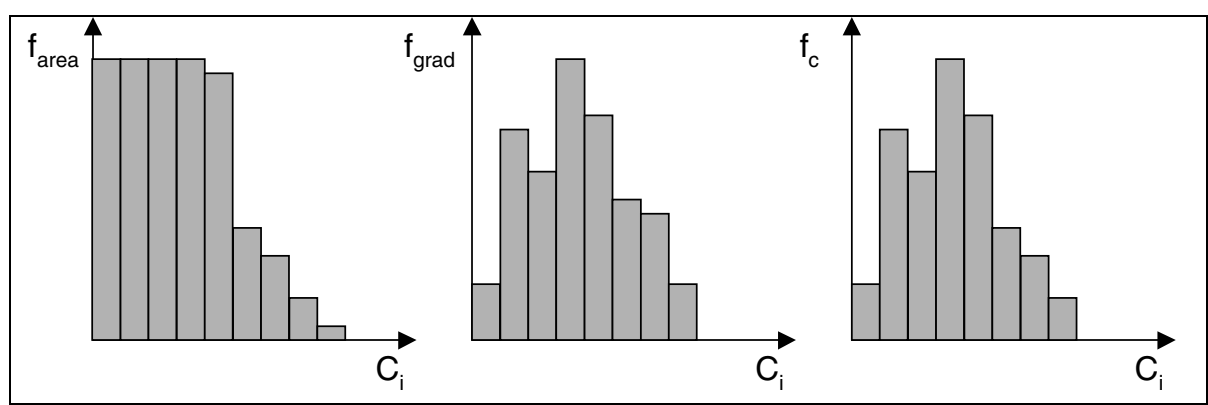

Fig. 4. Membership values for the fuzzy contour of the candidate particle described in Fig. 3 for different criteria: small area (left), large gradient under the contour (middle) using fuzzy sets in Fig. 2, and conjunction of both criteria representing the membership values to the class microcalcification contour (right) for a fuzzy contour corresponding to a single candidate particle 


\section{Partial Defuzzification}

We now build a fuzzy particle for each candidate particle, using the membership function to the microcalcification contour class of its respective fuzzy contour. This process will be called partial defuzzification, since it consists in defuzzifying some aspects of the fuzzy contours. To derive a very simple aggregation process in the $3 \mathrm{D}$ space of the particles detected on projected views, we transform the fuzzy contours into fuzzy particles in a two-step process.

First, for each candidate contour $C_{i}$ of a fuzzy contour with a membership function to the microcalcification contour class $f_{c}$, a connected component $C_{i}$ is created such as

$$
\forall(x, y) \in \dot{C}_{i}, \quad \dot{C}_{i}(x, y)=f_{c}\left(C_{i}\right)
$$

where $\dot{C}_{i}$ denotes the connected component that includes all pixels on or delimited by the contour $C_{i}$.

Then, for a projection image $P$, we generate a fuzzy particle map $I$ such that the value of each pixel is determined by

$$
\forall(x, y) \in P \quad I(x, y)=\vee_{i}\left[\dot{C}_{i}(x, y)\right]
$$

In summary, the aim of this partial defuzzification is to create a fuzzy particle map, where each pixel value corresponds to the possibility of that pixel belonging to a microcalcification. Since a single pixel may be enclosed by several different candidate contours, the membership values corresponding to each of these contours are combined in order to obtain the value for this pixel. The "max" operator is the smallest T-conorm and it realizes the equivalent of a union operation on fuzzy sets. It is used here to combine the different membership values corresponding to a given point.

\section{Aggregation and Final Decision}

After performing separate fuzzy detections in each of the $\mathrm{N}$ projection images of the DBT acquisition, the next step consists in aggregating the fuzzy particles by taking into account the acquisition geometry. The goal is to find for each $3 \mathrm{D}$ voxel the corresponding information in all of the $N$ fuzzy particle maps that were created.

The aggregation of information gathered in the fuzzy particle maps for a given voxel is expressed as

$$
I\left(x_{v}, y_{v}, z_{v}\right)=\stackrel{N}{\Psi}_{k=1}^{N}\left[I_{k}\left(x_{k}, y_{k}\right)\right]
$$

where $I\left(x_{v}, y_{v}, z_{v}\right)$ is the voxel intensity at position $\left(x_{v}, y_{v}, z_{v}\right), I\left(x_{k}, y_{k}\right)$ is the pixel intensity at position $\left(x_{k}, y_{k}\right)$ of the $k^{\text {th }}$ fuzzy particle map, corresponding to the 


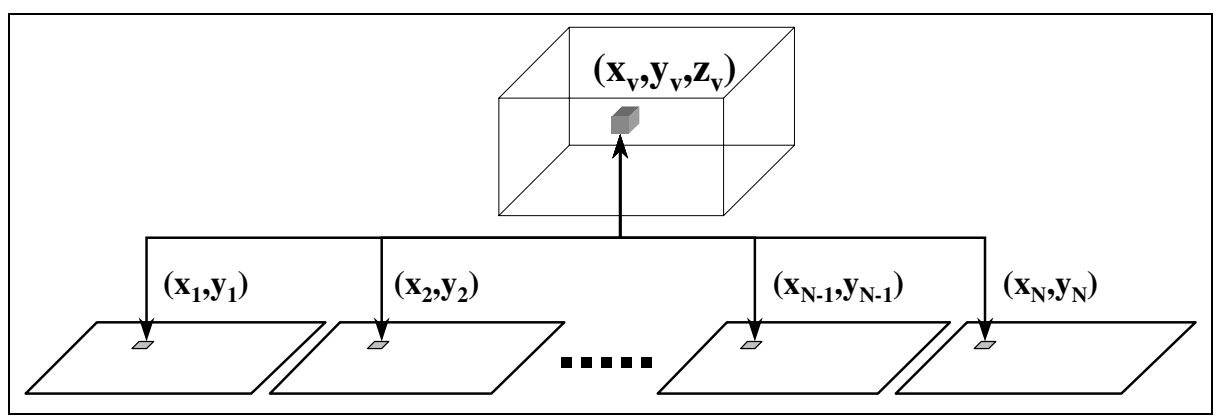

Fig. 5. Information aggregation strategy: For a given voxel $\left(x_{v}, y_{v}, z_{v}\right)$ the information from all corresponding pixels $\left(x_{k}, y_{k}\right)$ is aggregated using the operator $\Psi$. The position of the pixel $\left(x_{k}\right.$, $y_{k}$ ) corresponding to the projection of a given voxel $\left(x_{v}, y_{v}, z_{v}\right)$ is computed using a priori knowledge about the acquisition geometry.

projection of position $\left(x_{v}, y_{v}, z_{v}\right)$, and $\Psi$ is the aggregation operator. Fig. 5 illustrates this aggregation operation.

Using the arithmetic mean as aggregation operator, equation (7) can be rewritten as follows:

$$
I\left(x_{v}, y_{v}, z_{v}\right)=\frac{1}{N} \sum_{k=1}^{N} I_{k}\left(s_{z, k} \cdot x+\xi_{x, z, k}, s_{z, k} \cdot y+\xi_{y, z, k}\right)
$$

where $\xi_{x, z, k}$ and $\xi_{y, z, k}$ are the shift factors in $x$ and $y$ direction and $s_{z, k}$ is the scaling factor. These factors result from the acquisition geometry.

Finally, a defuzzification is applied to the 3D fuzzy particles, taking into account information acquired during the different processing steps, to decide whether particles correspond to microcalcifications. For reasons of simplicity, a simple thresholding was implemented as defuzzification in this preliminary approach.

\section{Preliminary Results}

In this section we show the result of applying these methods to real DBT data. Fig. 6 shows a projected view and corresponding fuzzy particle map. In Fig. 7 we see the results of aggregating in 3D before and after defuzzification (middle and right) alongside a reconstruction slice (left) that was reconstructed for comparison using Algebraic Reconstruction Technique (ART).

The validity of the proposed approach is illustrated in this example for a cluster of microcalcifications. Microcalcifications of different sizes, shapes and local contrast are detected. Since a clinical database providing ground truth at particle level is hard to come by, a visual sanity check today is the only means to verify our results. As 3D DBT datasets become increasingly numerous, a validation for detection of clusters of microcalcifications on a clinical database should be envisioned. 

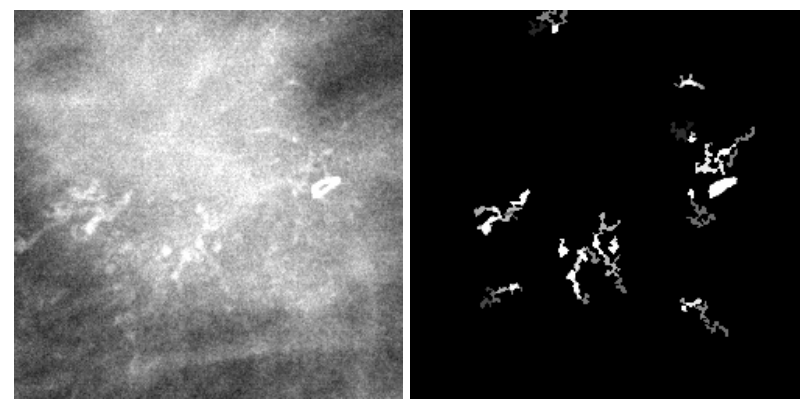

Fig. 6. Selected region of a DBT projected view (left) and the corresponding fuzzy particle map (right) (Tomographic projection data provided courtesy of Dr. D. Kopans, Massachusetts General Hospital, Boston, MA, USA.)
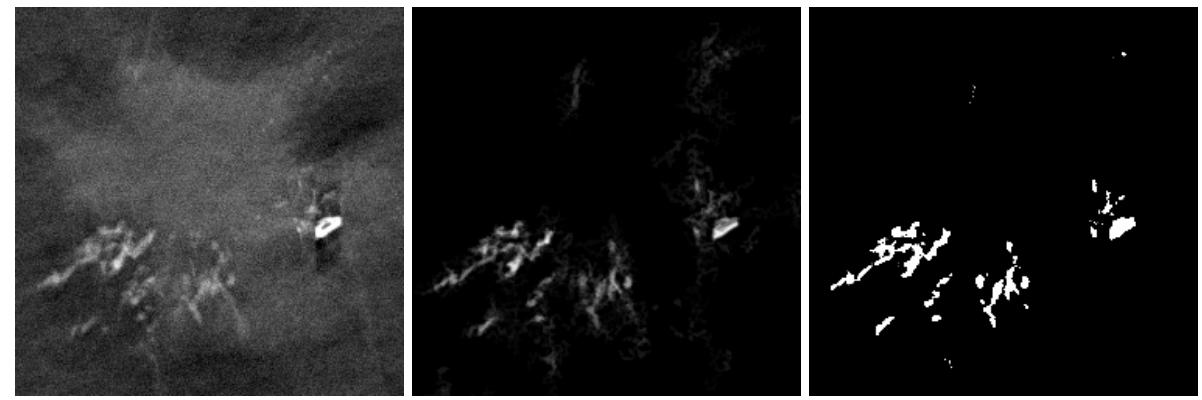

Fig. 7. Selected region of a slice reconstructed with ART (left), the corresponding 3D fuzzy particles in the same slice (middle), and corresponding 3D particles resulting from defuzzification of the 3D fuzzy particles by applying a threshold (right)

\section{Conclusion}

We have proposed a novel approach to detect microcalcifications in DBT datasets. Our approach exhibits numerous advantages. Working directly on the DBT projected views enables us to work independently of the reconstruction algorithm used to generate the $3 \mathrm{D}$ images. In addition, the processing time is expected to be significantly reduced compared to the application of similar operators on reconstructed slices, since they are generally much more numerous than the projected views, and the required $3 \mathrm{D}$ processing is sparse.

We have introduced a fuzzy description of the candidate particles to account for the ambiguities in the image data. Another key advantage of combining fuzzy techniques with a detection applied directly on the projected views is that information about each potential microcalcification can be preserved until the aggregation step. A final defuzzification of the aggregated particles allows the reduction of false positives that were accepted at a high level during the initial detection step in the projected views. 
The preliminary experiments presented in this paper are quite promising as far as a visual verification is concerned. Nevertheless, an investigation on a clinical database is needed for comparing detection results to state-of-the-art 2D detection algorithms.

\section{References}

1. K. Kerlikowske, H. Schipper, In: Fast Facts-Breast Cancer (Epidemiology), Health Press Limited, Oxford, UK, 1998.

2. T. Holland et al, So-called interval cancers of the breast: pathologic and radiographic analysis. Cancer 49, 2527-2533, 1982.

3. Dobbins III, J.T., Godfrey, D.J.: Digital x-ray tomosynthesis: current state of the art and clinical potential. Physics in Medicine and Biology 48, R65-R106, 2003.

4. Wu, T., Stewart, A., Stanton, M., McCauley, T., Phillips, W., Kopans, D.B., Moore, R.H., Eberhard, J.W., Opsahl-Ong, B., Niklason, L., Williams, M.B.: Tomo-graphic mammography using a limited number of low-dose cone-beam projection images. Medical Physics 30, 365-380, 2003.

5. M. Lanyi, In: Diagnosis and Differential Diagnosis of Breast Calcifications, SpringerVerlag Berlin Heidelberg, Germany, 1988.

6. H.-P. Chan, J. Wei, B. Sahiner, E.A. Rafferty, T. Wu, M.A. Roubidoux, R.H. Moore, D.B. Kopans, L.M. Hadjiiski, M.A. Helvie, Computerized Detection of Masses on Digital Tomosynthesis Mammograms - A preliminary Study. In: Proceedings of the 7th International Workshop on Digital Mammography, Chapel Hill, NC, Springer, 2004.

7. I. Reiser, R.M. Nishikawa, M.L. Giger, D.B. Kopans, E.A. Rafferty, R. Moore, T. Wu, A Reconstruction-Independent Method for Computerized Detection of Mammographic Masses in Tomosynthesis Images. In: Proceedings of the 7th International Workshop on Digital Mammography, Chapel Hill, NC, Springer, 2004.

8. H.D. Cheng, Y.M. Lui, R.I. Freimanis, A novel Approach to Microcalcification Detection Using Fuzzy Logic Technique. IEEE Transactions on Medical Imaging, 17 (3) 442-450, 1998.

9. S. Bothorel, B. Bouchon-Meunier, S. Muller, A Fuzzy Logic Based Approach for Semiological Analysis of Microcalcifications in Mammographic Images. International Journal for Intelligent Systems, 12, 819-848, 1997.

10. N. Pandey, Z. Salcic, J. Sivaswamy, Fuzzy Logic Based Microcalcification Detection. Neural Networks for Signal Processing - Proceedings of the IEEE Work-shop, 2 , 662-671, 2000. 